\title{
CONCENTRAÇÃO DA SOLUÇÃO DE TETRAZÓLIO E PERÍODO DE COLORAÇÃO DO TESTE PARA SEMENTES DE MAMONEIRA ${ }^{1}$
}

\author{
CAROLINA MARIA GASPAR-OLIVEIRA² ${ }^{2}$ CIBELE CHALITA MARTINS ${ }^{3}$, JOÃO NAKAGAWA ${ }^{4}$
}

\begin{abstract}
RESUMO - O objetivo do trabalho foi estudar a concentração da solução de tetrazólio e o período de coloração do teste para a avaliação do potencial fisiológico de sementes de mamoneira (Ricinus communis L.), padronizando a nomenclatura das cores observadas nas sementes após a coloração. Os tratamentos de concentração da solução de tetrazólio e períodos de coloração estudados foram: $0,075 \%$ e $0,1 \%$ por 120,180 e 240 minutos, $0,2 \%$ por 60,120 e 180 minutos, 0,5\% por 60, 90 e 120 minutos e 1,0\% por 30, 60 e 90 minutos. Os resultados foram comparados com os obtidos nos testes de germinação. A coloração das sementes após o teste de tetrazólio, em cada tratamento, foi avaliada mediante comparação com as fichas de cor do catálogo de Munsell, determinando-se a porcentagem de sementes observada em cada cor. O delineamento experimental foi o inteiramente casualizado e a comparação de médias realizada pelo teste de Tukey a 5\% de probabilidade. Para avaliar o potencial fisiológico pelo teste de tetrazólio, as sementes de mamoneira devem ser imersas na solução de tetrazólio na concentração de $0,2 \%$ por 120 minutos, a $35^{\circ} \mathrm{C}$. Nesse tratamento, as sementes viáveis após a coloração na solução de tetrazólio apresentaram predominantemente as cores rosa e rosa-escuro em suas estruturas essenciais, portanto essas podem ser consideradas como as cores características para o teste de tetrazólio em sementes de mamoneira.
\end{abstract}

Termos para indexação: viabilidade, mamona, metodologia, coloração.

\section{TETRAZOLIUM SOLUTION CONCENTRATION AND TEST STAINING PERIOD FOR CASTOR BEAN SEEDS}

\begin{abstract}
The objective of this research was to study the tetrazolium solution concentration and staining period for the evaluation of the physiological quality of castor bean seeds (Ricinus communis L.) by the tetrazolium test, standardizing the nomenclature of the observed colors in seeds after staining. The treatments of tetrazolium solution concentration and staining period were: $0.075 \%$ and $0.1 \%$ for 120,180 and 240 minutes, $0.2 \%$ for 60,120 and 180 minutes, $0.5 \%$ for 60,90 and 120 minutes and $1.0 \%$ for 30, 60 and 90 minutes. The results were compared to the germination test. The seed color after the tetrazolium test in each treatment was evaluated by comparing with the Munsell book of color, and the percentage of seeds observed in each color was established. A randomized complete block design was used and the means were compared by the Tukey test at the 0.05 level of probability. To evaluate physiological quality by the tetrazolium test, castor bean seeds should be placed in tetrazolium solution at the concentration $0.2 \%$ for 120 minutes, at $35^{\circ} \mathrm{C}$, for staining development, and in this treatment, the viable seeds after the staining in tetrazolium solution showed
\end{abstract}

${ }^{1}$ Submetido em 26/03/2008. Aceito para publicação em16/09/2009. Parte da Tese de Doutorado do primeiro autor apresentada a UNESP/Faculdade de Ciências Agronômicas/Campus Botucatu.

²Eng. Agr. Doutora, Bolsista pós-doutorado júnior CNPq, Embrapa-Soja, Londrina-PR, Caixa Postal 231, Cep: 86001-970-cmgasparol@gmail.com

${ }^{3}$ Professora colaboradora, Departamento de Produção Vegetal/Agricultura,
FCA/UNESP, Caixa postal 237-CEP: 18610-307, Botucatu-SP, E-mail: cibele@fca.unesp.br

${ }^{4}$ Professor Titular aposentado, voluntário, Departamento de Produção Vegetal/Agricultura, FCA/UNESP, Caixa postal 237, CEP: 18610-307Botucatu-SP, secdamv@fca.unesp.br 
predominantly the colors pink and dark pink in their essential structures. Thus these colors can be considered as the characteristics colors for the tetrazolium test in castor bean seeds.

Index terms: viability, methodology, color.

\section{INTRODUÇÃO}

A eficiência do teste de tetrazólio em avaliar a viabilidade e, em alguns casos, o vigor das sementes está relacionada ao desenvolvimento de métodos apropriados e ao estabelecimento de critérios complementares para a avaliação de cada espécie, como foi realizado para seringueira (Wetzel et al., 1992), amendoim (Bittencourt e Vieira, 1997), soja (França Neto et al., 1998), milho (Dias e Barros, 1999), algodão (Vieira e Von Pinho, 1999), tomate (Santos et al., 2007), abobrinha (Barros et al., 2005), melancia (Bhering et al., 2005), entre outras espécies.

Em sementes de mamoneira (Ricinus communis L.) o teste de tetrazólio ainda não tem uso generalizado, principalmente, devido à deficiência de conhecimentos sobre a metodologia adequada, já que as recomendações encontradas para a realização desse teste são restritas e imprecisas em função da carência de pesquisas relacionadas ao assunto. Assim, as diferenças entre os métodos sugeridos acarretam dificuldades para o estabelecimento de padrões a serem seguidos, como pode se observar nas Regras para Análise de Sementes (Brasil, 1992), na qual a recomendação para sementes de mamoneira é a coloração na solução de tetrazólio a $1,0 \%$, à temperatura de $30^{\circ} \mathrm{C}$, e por um período de seis a 24 horas. Diferente da prescrição de Grabe (1976), também para essa espécie, que recomenda concentração da solução de tetrazólio de $1,0 \%$ por duas a três horas, a $35^{\circ} \mathrm{C}$.

O período, a temperatura e a concentração da solução de tetrazólio dependem das características de cada espécie e, geralmente, são determinados a partir de estudos que comparam os resultados obtidos no teste de tetrazólio com outros testes que avaliam a qualidade das sementes, como a germinação e a emergência de plântulas (Wetzel et al., 1992; Barros et al., 2005; Bhering et al., 2005). A definição desses parâmetros para cada espécie é importante, pois eles influenciam diretamente a intensidade e a uniformidade de coloração das sementes, ou seja, interferem na avaliação e na interpretação dos resultados do teste de tetrazólio, podendo alterá-los.

Wetzel et al. (1992) recomendaram a coloração de sementes de seringueira em solução de tetrazólio a $0,5 \%$ por duas a três horas, a $40^{\circ} \mathrm{C}$. Esses autores verificaram que para essa espécie quanto maior o período de pré-condicionamento, menor o período de coloração e vice-versa. Em sementes de soja, a concentração da solução utilizada é de $0,075 \%$ e temperatura de 35 a $40^{\circ} \mathrm{C}$, por 150 a 180 minutos (França Neto et al., 1998). Bittencourt e Vieira (1997) recomendaram a exposição das sementes de amendoim sem casca em solução $0,05 \%$ de tetrazólio por três horas, a $40^{\circ} \mathrm{C}$. Para algodão utiliza-se solução de tetrazólio a $0,1 \%$, a $30^{\circ} \mathrm{C}$ por aproximadamente quatro horas (Vieira e Von Pinho, 1999).

Várias concentrações da solução de tetrazólio podem ser utilizadas no teste, dependendo da espécie avaliada, do método de preparo das sementes e da permeabilidade da casca, sendo as mais utilizadas $0,075 \%, 0,1 \%, 0,2 \%, 0,5 \%$ e 1,0\% (Delouche et al., 1976; Marcos Filho et al., 1987). Entretanto, são recomendadas as menores concentrações do sal por possibilitarem melhor visualização da coloração dos tecidos e dos diferentes tipos de injúrias (Marcos Filho et al., 1987; França Neto et al., 1998). O período de coloração depende da temperatura, da concentração da solução e da espécie em análise, mas geralmente encontra-se entre 30 e 240 minutos (Delouche et al., 1976; Marcos Filho et al., 1987).

A precisão do teste de tetrazólio não é afetada por temperaturas entre 20 e $45^{\circ} \mathrm{C}$, mas a coloração se estabelece mais rapidamente nas temperaturas mais elevadas (Grabe, 1976). Por esse motivo, Marcos Filho et al. (1987) recomendaram que as sementes imersas na solução de tetrazólio sejam colocadas em câmara regulada à temperatura de 30 a $40^{\circ} \mathrm{C}$, e mantidas no escuro, pois a solução de tetrazólio é fotossensível, e a luz pode alterar a coloração e comprometer os resultados do teste.

As diferenças de cor observadas nas sementes após a coloração na solução de tetrazólio são as principais características que devem ser consideradas na interpretação dos resultados do teste. A intensidade de coloração das sementes no teste de tetrazólio é variável entre as espécies. Por exemplo, a cor rosa observada em sementes viáveis e de alto vigor de soja (França Neto et al., 1998), é mais clara que a verificada em sementes de mesma qualidade de milho (Dias e Barros, 1999) e algodão (Vieira e Von Pinho, 1999). Nessas espécies a cor que sinaliza um tecido de alto vigor é a rosa-escuro ou vermelha. Em sementes de soja essa cor 
rosa-escuro significaria tecido em deterioração. Isso ocorre, pois a terminologia utilizada para denominar as cores observadas nas sementes no teste de tetrazólio costuma ser estabelecida pelos autores, e por isso pode variar entre os trabalhos. Sementes de soja e de amendoim apresentam tonalidades de coloração semelhantes, entretanto, de acordo com Bittencourt e Vieira (1997), em amendoim a cor rosa suave designa tecidos vigorosos, as cores rosa muito intenso e vermelho intenso indicam tecido em deterioração, e as cores roxas e os vermelhos muito intensos indicam tecidos muito deteriorados. Para sementes de soja, França Neto et al. (1998) descreveram a cor vermelho carmim para tecido vivo e vigoroso, e a cor vermelho carmim forte ou vermelho intenso para tecidos em deterioração.

Essas diferenças podem acarretar problemas de interpretação durante a avaliação das sementes. Por essa razão é interessante utilizar-se de uma terminologia padronizada, correlacionando-se as cores observadas nas sementes com uma referência não subjetiva. Nesse contexto, o catálogo de cores de Munsell (Munsell, 1976) apresentase como um padrão de classificação de cores, sendo muito utilizado em diversas áreas da ciência como na classificação de solos, descrição de cores de frutos, alimentos e bebidas, fabricação de tintas, desenhos gráficos, e outros.

Portanto, o trabalho foi realizado com o objetivo de estudar a concentração da solução de tetrazólio e período de coloração do teste para a avaliação do potencial fisiológico de sementes de mamoneira, padronizando a nomenclatura das cores observadas nas sementes após a coloração.

\section{MATERIAL E MÉTODOS}

Neste trabalho foram utilizados cinco lotes de sementes de mamoneira, cultivar AL Guarany 2002, de diversas procedências, numerados de 1 a 5 , homogeneizados e amostrados em $2.000 \mathrm{~g}$ cada um, para a realização das análises (Brasil, 1992).

A caracterização da qualidade física e fisiológica dos lotes de sementes foi realizada pelos testes:

Teor de água, determinado pelo método da estufa a $105 \pm 3^{\circ} \mathrm{C}$ por 24 horas (Brasil, 1992), utilizando duas subamostras de 15 sementes.

Teste de germinação em areia, realizado com oito subamostras de 25 sementes, semeadas entre areia esterilizada (EA), previamente umedecida com água destilada, na proporção de $50 \%$ da capacidade de retenção (Brasil, 1992). Conduzido dentro de caixas de plástico transparente $(110 \times 110 \times 35 \mathrm{~mm})$, sendo utilizadas duas por subamostra, acondicionadas em sacos de plástico $(0,033 \mathrm{~mm}$ de espessura) para a manutenção da umidade, sob alternância de temperatura $\left(20^{\circ} \mathrm{C} / 16\right.$ horas e $30^{\circ} \mathrm{C} / 8$ horas sob luz). No $14^{\circ}$ dia após a instalação do teste, foram contabilizadas as plântulas normais, anormais e as sementes mortas e duras (Brasil, 1992). As sementes duras foram escarificadas com lixa d'água número 80 , e colocadas para germinar por mais sete dias, em rolo de papel (RP), umedecido com 2,5 vezes a massa ( $\mathrm{g}$ ) do papel em água destilada, sob mesma alternância de temperatura, para verificar a sua viabilidade.

No teste de germinação em papel foram semeadas oito subamostras de 25 sementes, em rolos de papel toalha para germinação (RP), previamente umedecidos com 2,5 vezes a massa ( $\mathrm{g}$ ) do papel em água destilada, acondicionados em sacos de plástico $(0,033 \mathrm{~mm}$ de espessura) para a manutenção da umidade (Brasil, 1992; Coimbra et al., 2007; Gaspar Oliveira et al., 2007). Os rolos foram dispostos na posição horizontal em germinador, sob temperaturas alternadas $\left(20^{\circ} \mathrm{C} / 16\right.$ horas e $30^{\circ} \mathrm{C} / 8$ horas sob luz), realizando-se a primeira contagem e a retirada das plântulas normais aos sete dias após a semeadura (Brasil, 1992). No 14 dia após a instalação do teste contabilizaram-se as plântulas normais, anormais e sementes mortas e duras. A viabilidade das sementes duras foi verificada adotando-se o mesmo procedimento descrito no teste de germinação em areia.

Na emergência de plântulas em campo foram utilizadas quatro subamostras de 50 sementes, semeadas diretamente no solo, a $5 \mathrm{~cm}$ de profundidade, no espaçamento de 20 cm entre linhas, dentro de túnel plástico, com irrigação controlada. Consideraram-se como plântulas emersas aquelas em que o cotilédone saiu do solo e estava aberto, aos 21 dias após a semeadura. Em conjunto com esse teste foi conduzido o índice de velocidade de emergência de plântulas (IVE), contabilizando-se diariamente o número de plântulas emersas até os 21 dias após a semeadura, e calculando-se o IVE pela fórmula proposta por Maguire (1962).

Baseando-se em resultados observados em Gaspar Oliveira (2007), foram testados os seguintes tratamentos de concentração da solução e período de coloração: 0,075\% e $0,1 \%$ por 120,180 e 240 minutos, $0,2 \%$ por 60,120 e 180 minutos, $0,5 \%$ por 60 , 90 e 120 minutos e $1,0 \%$ por 30,60 e 90 minutos.

Em cada tratamento avaliado, quatro subamostras de 25 sementes foram pré-condicionadas entre papel toalha de germinação, umedecido com 2,5 vezes a massa $(\mathrm{g})$ do papel em água destilada, dobrado em quatro e colocado 
dentro de sacos de plástico $(0,05 \mathrm{~mm}$ de espessura) para manter a umidade, por 18 horas a $30^{\circ} \mathrm{C}$ (Moore, 1985; Brasil, 1992; França Neto et al., 1998). Após esse período, a casca foi removida e as sementes cortadas longitudinal e medianamente, no sentido do comprimento, através do endosperma e embrião, selecionando-se a melhor metade de cada uma delas (Gaspar Oliveira et al., 2009). As amostras foram colocadas em copos plásticos, imersas na solução de tetrazólio, e mantidas em câmara de germinação a $35^{\circ} \mathrm{C}$, na ausência de luz. Após a coloração, a solução de tetrazólio foi drenada e as sementes lavadas em água corrente para estacionar o processo.

As sementes foram avaliadas uma a uma e classificadas em viáveis e não viáveis. Os critérios para a interpretação da viabilidade das sementes foram baseados no estudo das partes vitais do embrião, na semelhança das estruturas essenciais desenvolvidas normalmente durante a germinação, na tonalidade e uniformidade da coloração, nas recomendações existentes para sementes de mamoneira (Grabe, 1976; Brasil, 1992), e na comparação dos testes de tetrazólio já estabelecidos para outras espécies de dicotiledôneas (Wetzel et al., 1992; Bittencourt e Vieira, 1997; França Neto et al., 1998; Vieira e Von Pinho, 1999).

As estruturas observadas nas sementes de mamoneira no teste de tetrazólio foram: o endosperma, a área de ligação entre os cotilédones e o eixo embrionário, o eixo hipocótiloradícula e os cotilédones. Os critérios para interpretar os resultados do teste de tetrazólio consideraram as sementes viáveis aquelas com estruturas do embrião bem desenvolvidas, intactas e com cor rosa a vermelha após a exposição ao sal de tetrazólio; com pequenas necroses nos cotilédones, ou em outras áreas que não na junção do eixo embrionário e dos cotilédones, ou na ponta extrema da radícula; com menos da metade da área do endosperma não colorida. As sementes não viáveis apresentavam ausência de coloração nos cotilédones e/ou eixo hipocótilo-radícula ou mais da metade das áreas do endosperma e embrião sem coloração (Figura 1).

A coloração das sementes após o teste de tetrazólio, em cada tratamento, foi avaliada mediante comparação com as fichas de cor do catálogo de Munsell (Munsell, 1976), determinando-se a porcentagem de sementes observada em cada cor. Depois, as cores observadas para as sementes de mamoneira após o teste foram, subjetivamente, renomeadas e classificadas nos grupos de cores rosa-pálido, rosa-claro, rosa, rosa-escuro, vermelho-carmim e vermelho-carmim escuro, pois essas denominações são mais usuais na literatura sobre o teste de tetrazólio (Delouche et al., 1976; Grabe, 1976; França Neto et al., 1998).
Os resultados de viabilidade do teste de tetrazólio, para cada tratamento, foram comparados com os resultados dos testes de germinação em areia e em papel, e foram considerados os melhores tratamentos aqueles que apresentaram diferença de até $5 \%$ entre os resultados dos testes (Dias e Barros, 1995; França Neto, 1999). Ao final foi considerado como o melhor tratamento aquele que proporcionou para a média dos lotes a melhor correlação entre os resultados do teste de tetrazólio e os testes de germinação em areia e em papel. Havendo mais de um tratamento nessas condições, optou-se por aquele que apresentou o menor período de coloração e/ou a menor concentração do sal de tetrazólio.

$\mathrm{Na}$ análise estatística utilizou-se o delineamento inteiramente casualizado, com comparação de médias pelo teste de Tukey a $5 \%$ de probabilidade (Banzatto e Kronka, 2006). Nos testes de germinação em que se utilizaram oito subamostras de 25 sementes, as mesmas foram agrupadas antes das avaliações, formando quatro repetições de 50 sementes. Na comparação dos resultados de viabilidade do teste de tetrazólio, conduzido nos tratamentos de concentração da solução e período de coloração (15 tratamentos e cinco lotes; quatro repetições), e da germinação em areia e em papel (cinco lotes; quatro repetições), as médias foram comparadas considerando cada lote separadamente, e também a média dos lotes. Realizouse também a análise de correlação simples (r) entre os dados dos testes de tetrazólio, germinação em areia e em papel. $\mathrm{Na}$ análise das cores das sementes as médias dos cinco lotes foram comparadas para os tratamentos do teste de tetrazólio (15 tratamentos e seis grupos de cores), e os dados foram transformados em arcsen raiz ( $\mathrm{x}+\mathrm{alfa}$ ) (Banzatto e Kronka, 2006), sendo que as médias apresentadas nas tabelas referem-se aos dados originais.

\section{RESULTADOS E DISCUSSÃO}

$\mathrm{Na}$ caracterização da qualidade física e fisiológica dos lotes de sementes de mamoneira todos os lotes apresentaram o mínimo de germinação estabelecido para a comercialização que é de $80 \%$, e teor de água semelhante, garantindo que os testes de avaliação de qualidade foram isentos de possíveis variações de resultados entre os lotes devido ao teor de água (Tabela 1). Foram verificadas diferenças de qualidade entre os lotes nos testes de germinação em areia, emergência de plântulas em campo e índice de velocidade de emergência (IVE), sendo que os lotes 4 e 5 tinham qualidade inferior aos demais lotes. 


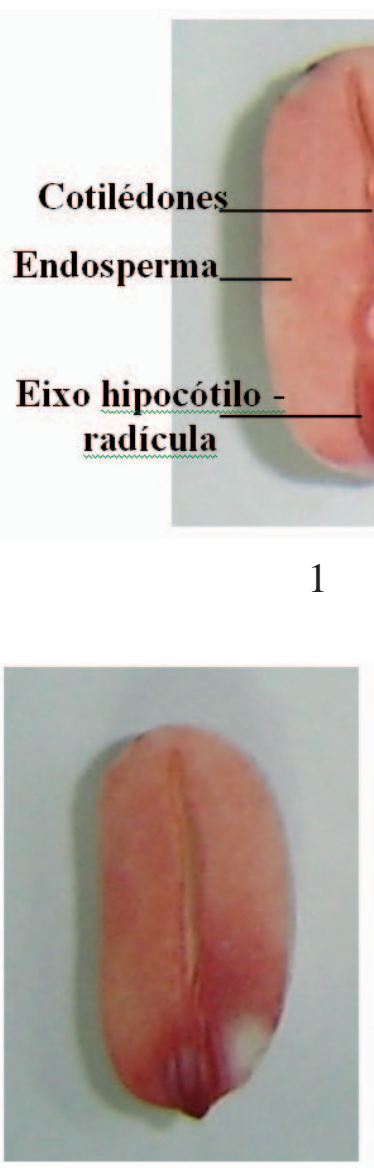

5

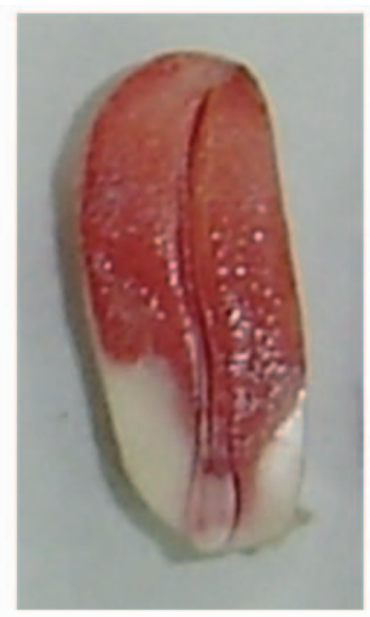

10

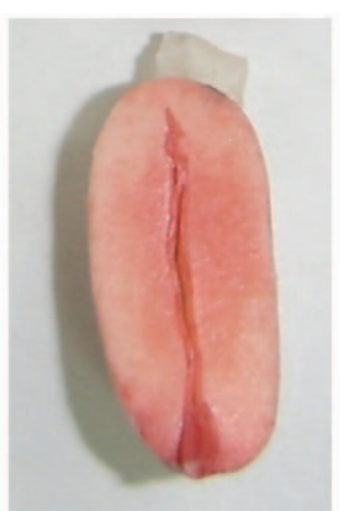

6



11



$3^{\wedge}$

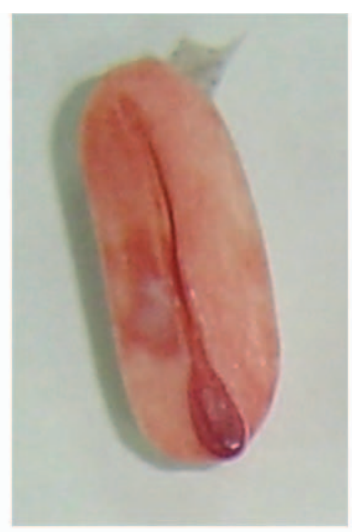

4



7

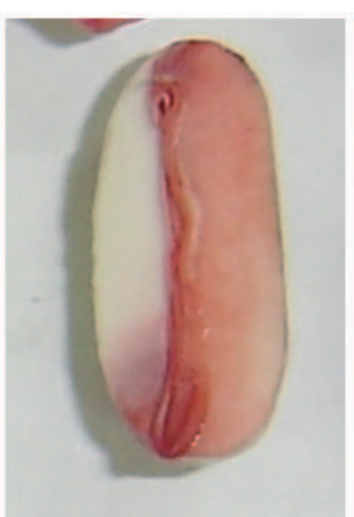

8

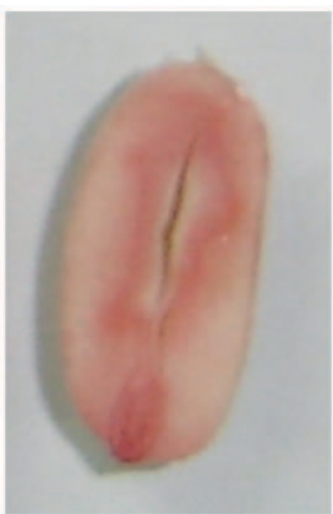

9



12

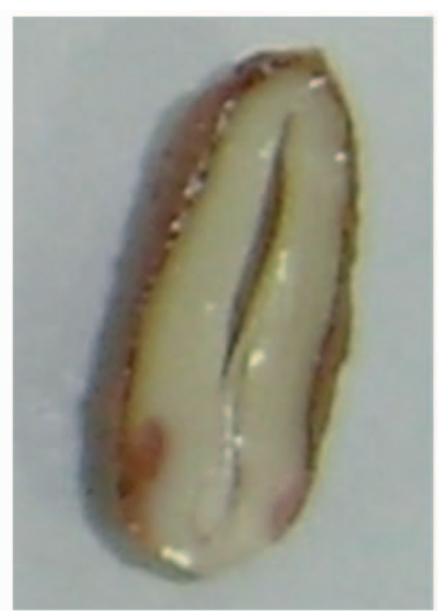

13

FIGURA 1. Sementes de mamoneira após o teste de tetrazólio. 1-8 Sementes viáveis: (1 - estruturas do embrião bem desenvolvidas, intactas e com cor rosa a vermelha; 2, 3 - pequenas necroses nos cotilédones; 4, 5 pequenas necroses em outras áreas que não na junção do eixo embrionário e dos cotilédones; 6 - pequenas necroses na ponta extrema da radícula; 7, 8 - menos da metade da área do endosperma não colorida); 9-13 Sementes não viáveis: $(9,10,11$ - ausência de coloração nos cotilédones e/ou eixo hipocótilo-radícula; 12, 13 - mais da metade das áreas do endosperma e embrião sem coloração). 
TABELA 1. Caracterização física e fisiológica dos cinco lotes de sementes de mamoneira, utilizados na segunda etapa, determinada pelos testes de teor de água (\%), germinação em areia (\%), germinação em papel (\%), emergência de plântulas em campo (\%) e índice de velocidade de emergência (IVE).

\begin{tabular}{cccccc}
\hline Lotes & Teor de água (\%) & $\begin{array}{c}\text { Germinação em } \\
\text { areia (\%) }\end{array}$ & $\begin{array}{c}\text { Germinação em } \\
\text { papel (\%) }\end{array}$ & $\begin{array}{c}\text { Emergência de } \\
\text { plântulas (\%) }\end{array}$ & IVE \\
\hline 1 & 5,0 & $98 \mathrm{~A}^{1}$ & 100 & $78 \mathrm{BC}$ & $4,1 \mathrm{~B}$ \\
2 & 5,5 & $99 \mathrm{~A}$ & 97 & $91 \mathrm{~A}$ & $4,8 \mathrm{~A}$ \\
3 & 5,0 & $98 \mathrm{~A}$ & 98 & $90 \mathrm{AB}$ & $4,8 \mathrm{~A}$ \\
4 & 5,0 & $91 \mathrm{AB}$ & 96 & $80 \mathrm{ABC}$ & $3,9 \mathrm{~B}$ \\
5 & 5,5 & $86 \mathrm{~B}$ & 94 & $74 \mathrm{C}$ & $3,9 \mathrm{~B}$ \\
\hline CV\% & 8,6 & 4,7 & 3,1 & 6,9 & 8,3 \\
DMS & 1,8 & 9,7 & 1,5 & 12,4 & 0,8 \\
\hline
\end{tabular}

A partir da análise de comparação de médias, realizada para cada lote separadamente (Tabela 2), verificou-se que não houve diferença estatística significativa entre os resultados dos testes de tetrazólio e de germinação para os lotes 1 e 5 . Nos demais lotes, diferenças na porcentagem de sementes viáveis entre os testes foram observadas. Destacou-se nos lotes 2 e 3 o tratamento $1,0 \%$ por 90 minutos, e no lote 4 os tratamentos $0,5 \%$ por 90 e 120 minutos, e $1,0 \%$ por 60 e 90 minutos como os que apresentaram os resultados mais discrepantes dos observados nos testes de germinação. Portanto, esses tratamentos não devem ser utilizados na condução do teste de tetrazólio em sementes de mamoneira, pois resultaram na coloração excessiva das sementes, mesmo as pouco vigorosas e, por isso, os resultados diferiram tanto dos observados no teste de germinação. Wetzel et al. (1992) também verificaram problemas de avaliação do teste de tetrazólio na concentração da solução de 1,0\% para sementes de seringueira.

Comparando-se as médias dos lotes, observou-se que ocorreram diferenças entre os testes de tetrazólio e os testes de germinação, entretanto apenas o tratamento 1,0\% por 60 minutos mostrou resultado menor estatisticamente; os demais foram iguais ou intermediários (Tabela 2).

A partir da classificação subjetiva das cores do catálogo de Munsell, observadas nas sementes de mamoneira após o teste de tetrazólio, nos grupos de cores usuais na literatura sobre o teste, e da análise de variância entre os grupos de cores e os tratamentos de concentração da solução e período de coloração, observou-se que houve diferença estatística significativa entre os grupos de cores para todos os tratamentos (Tabela 3 ).

Dentre eles, destacaram-se os tratamentos considerados os melhores na comparação com os resultados de viabilidade pelo teste de tetrazólio (Tabela 2). Assim, de forma geral, nos tratamentos com as concentrações de $0,075 \%$ e de $0,1 \%$, em todos os períodos, e de $0,2 \%$ por 60 e 120 minutos a maior parte das sementes apresentou-se nos grupos de cores rosa-claro, rosa e rosa-escuro (Tabela $3)$. Nos tratamentos de concentração da solução de $0,2 \%$ por 180 minutos, de $0,5 \%$ por 60 minutos e de $1,0 \%$ por 30 minutos a maioria das sementes apresentou-se nos grupos de cores rosa, rosa-escuro e vermelho carmim.

As cores mais escuras nas sementes refletiram o aumento gradativo da concentração da solução de tetrazólio e do período de coloração, e quanto mais escura a cor da semente, maior foi a dificuldade de visualização dos tecidos e a identificação das injúrias, podendo confundir tecidos vivos com aqueles em deterioração (Marcos Filho et al, 1987). Como ocorreu nos tratamentos de concentração $0,5 \%$ por 120 minutos e $1,0 \%$ por 60 e 90 minutos, para os quais a cor mais escura apresentada pelas sementes resultou na dificuldade de avaliação da viabilidade, contribuindo para que esses tratamentos apresentassem resultados do teste de tetrazólio muito discrepantes dos observados nos testes de germinação (Tabela 2). 
TABELA 2. Sementes de mamoneira viáveis pelos testes de tetrazólio (TZ) conduzidos nos diferentes tratamentos de concentração da solução e período de coloração, e pelos testes de germinação em areia e em papel.

\begin{tabular}{|c|c|c|c|c|c|c|c|c|}
\hline \multirow{2}{*}{\multicolumn{3}{|c|}{ Tratamentos }} & \multicolumn{6}{|c|}{ Sementes viáveis (\%) } \\
\hline & & & \multirow{2}{*}{$\begin{array}{c}\text { Lote } 1 \\
98\end{array}$} & \multirow{2}{*}{$\begin{array}{c}\text { Lote } 2 \\
99 \mathrm{~A}\end{array}$} & \multirow{2}{*}{$\begin{array}{c}\text { Lote } 3 \\
98 \mathrm{~A}\end{array}$} & \multirow{2}{*}{$\begin{array}{l}\text { Lote } 4 \\
91 \mathrm{AB}\end{array}$} & \multirow{2}{*}{$\begin{array}{c}\text { Lote } 5 \\
86\end{array}$} & \multirow{2}{*}{$\frac{\text { Média dos lotes }}{94 \mathrm{~A}^{1}}$} \\
\hline & Germinação em & areia & & & & & & \\
\hline & Germinação em & papel & 100 & $97 \mathrm{~A}$ & $98 \mathrm{~A}$ & $96 \mathrm{~A}$ & 94 & $97 \mathrm{~A}$ \\
\hline & $0,075 \%$ & x $120 \mathrm{~min}$ & 93 & $93 \mathrm{~A}$ & $88 \mathrm{AB}$ & $93 \mathrm{~A}$ & 95 & $92 \mathrm{AB}$ \\
\hline \multirow[t]{3}{*}{$\mathrm{TZ}$} & & x $180 \min$ & 98 & $98 \mathrm{~A}$ & $97 \mathrm{~A}$ & $98 \mathrm{~A}$ & 92 & $97 \mathrm{~A}$ \\
\hline & & x $240 \min$ & 97 & $98 \mathrm{~A}$ & $93 \mathrm{AB}$ & $94 \mathrm{~A}$ & 90 & $94 \mathrm{~A}$ \\
\hline & $0,1 \%$ & x $120 \min$ & 94 & $93 \mathrm{~A}$ & $88 \mathrm{AB}$ & $98 \mathrm{~A}$ & 88 & $92 \mathrm{AB}$ \\
\hline \multirow[t]{3}{*}{$\mathrm{TZ}$} & & x $180 \min$ & 99 & $92 \mathrm{~A}$ & $96 \mathrm{~A}$ & $97 \mathrm{~A}$ & 93 & $95 \mathrm{~A}$ \\
\hline & & x $240 \min$ & 97 & $95 \mathrm{~A}$ & $93 \mathrm{AB}$ & $98 \mathrm{~A}$ & 94 & $95 \mathrm{~A}$ \\
\hline & $0,2 \%$ & $\mathrm{x} \quad 60 \mathrm{~min}$ & 99 & $94 \mathrm{~A}$ & $95 \mathrm{~A}$ & $95 \mathrm{~A}$ & 89 & $94 \mathrm{~A}$ \\
\hline \multirow[t]{3}{*}{$\mathrm{TZ}$} & & x $120 \min$ & 98 & $94 \mathrm{~A}$ & $94 \mathrm{AB}$ & $92 \mathrm{~A}$ & 91 & $94 \mathrm{~A}$ \\
\hline & & x $180 \min$ & 97 & $89 \mathrm{~A}$ & $91 \mathrm{AB}$ & $96 \mathrm{~A}$ & 93 & $93 \mathrm{~A}$ \\
\hline & $0,5 \%$ & $x \quad 60 \min$ & 99 & $91 \mathrm{~A}$ & $90 \mathrm{AB}$ & $90 \mathrm{AB}$ & 92 & $92 \mathrm{AB}$ \\
\hline \multirow[t]{3}{*}{$\mathrm{TZ}$} & & $\mathrm{x} \quad 90 \mathrm{~min}$ & 100 & $74 \mathrm{AB}$ & $87 \mathrm{AB}$ & $81 \mathrm{BC}$ & 91 & $87 \mathrm{AB}$ \\
\hline & & $\mathrm{x} 120 \mathrm{~min}$ & 99 & $83 \mathrm{~A}$ & $89 \mathrm{AB}$ & $79 \mathrm{C}$ & 87 & $87 \mathrm{AB}$ \\
\hline & $1,0 \%$ & $x \quad 30 \min$ & 97 & $85 \mathrm{~A}$ & $87 \mathrm{AB}$ & $97 \mathrm{~A}$ & 96 & $92 \mathrm{AB}$ \\
\hline \multirow[t]{4}{*}{$\mathrm{TZ}$} & & $x \quad 60 \min$ & 96 & $75 \mathrm{AB}$ & $79 \mathrm{AB}$ & $77 \mathrm{C}$ & 85 & $82 \mathrm{~B}$ \\
\hline & & $\mathrm{x} \quad 90 \mathrm{~min}$ & 96 & $47 \mathrm{~B}$ & $75 \mathrm{~B}$ & $79 \mathrm{C}$ & 89 & $94 \mathrm{~A}$ \\
\hline & $\mathrm{CV} \%$ & & 2,0 & 8,0 & 5,3 & 2,7 & 3,4 & 6,0 \\
\hline & DMS & & 7,9 & 28,3 & 19,4 & 10,0 & 12,4 & 10,8 \\
\hline
\end{tabular}


TABELA 3. Porcentagem das sementes de mamoneira distribuídas nos grupos de cores, com as correspondentes cores do catálogo de Munsell, após teste de tetrazólio conduzido nos diferentes tratamentos de concentração da solução (\%) e período de coloração (minutos).

\begin{tabular}{|c|c|c|c|c|c|c|c|c|c|c|c|c|c|c|c|c|}
\hline \multirow{3}{*}{$\begin{array}{l}\text { Grupos de } \\
\text { cores }\end{array}$} & \multirow{3}{*}{$\begin{array}{l}\text { Cores do } \\
\text { catálogo de } \\
\text { Munsell }\end{array}$} & & & & \multicolumn{12}{|c|}{ Tratamentos do teste de tetrazólio } \\
\hline & & \multicolumn{3}{|c|}{$0,075 \%$} & \multicolumn{3}{|c|}{$0,1 \%$} & \multicolumn{3}{|c|}{$0,2 \%$} & \multicolumn{3}{|c|}{$0,5 \%$} & \multicolumn{3}{|c|}{$1,0 \%$} \\
\hline & & 120 & 180 & 240 & 120 & 180 & 240 & 60 & 120 & 180 & 60 & 90 & 120 & 30 & 60 & 90 \\
\hline Rosa-pálido & $2,5 \mathrm{R} 9 / 2$ & $1 \mathrm{C}$ & $1 \mathrm{C}$ & $1 \mathrm{C}$ & $1 \mathrm{C}$ & $0 \mathrm{~B}$ & $0 \mathrm{D}$ & $1 \mathrm{C}$ & $0 \mathrm{C}$ & $0 \mathrm{C}$ & $0 \mathrm{D}$ & $1 \mathrm{C}$ & $1 \mathrm{C}$ & $\begin{array}{l}0 \\
\mathrm{~B}\end{array}$ & $0 \mathrm{C}$ & $1 \mathrm{C}$ \\
\hline Rosa-claro & $\begin{array}{l}5 \mathrm{R} \quad 8 / 4 \\
5 \mathrm{R} \quad 8 / 6 \\
2,5 \mathrm{R} 8 / 6\end{array}$ & $60 \mathrm{~A}$ & $36 \mathrm{~A}$ & $28 \mathrm{~B}$ & $42 \mathrm{~A}$ & $34 \mathrm{~A}$ & $17 \mathrm{~B}$ & $26 \mathrm{~B}$ & $15 \mathrm{~B}$ & $2 \mathrm{C}$ & $1 \mathrm{D}$ & $1 \mathrm{C}$ & $0 \mathrm{C}$ & $\begin{array}{l}1 \\
\mathrm{~B}\end{array}$ & $0 \mathrm{C}$ & $1 \mathrm{C}$ \\
\hline Rosa & $\begin{array}{l}2,5 \mathrm{R} 7 / 6 \\
2,5 \mathrm{R} 7 / 8 \\
2,5 \mathrm{R} 6 / 10\end{array}$ & $23 \mathrm{~B}$ & $42 \mathrm{~A}$ & $44 \mathrm{~A}$ & $37 \mathrm{~A}$ & $37 \mathrm{~A}$ & $39 \mathrm{~A}$ & $44 \mathrm{~A}$ & $41 \mathrm{~A}$ & $21 \mathrm{~B}$ & $28 \mathrm{~B}$ & $10 \mathrm{~B}$ & $11 \mathrm{~B}$ & $26 \mathrm{~A}$ & A $6 \mathrm{~B}$ & $1 \mathrm{C}$ \\
\hline Rosa-escuro & $\begin{array}{c}2,5 \mathrm{R} 6 / 12 \\
1,25 \mathrm{R} 6 / 12\end{array}$ & $14 \mathrm{~B}$ & $19 \mathrm{~B}$ & $24 \mathrm{~B}$ & $18 \mathrm{~B}$ & $26 \mathrm{~A}$ & $39 \mathrm{~A}$ & $25 \mathrm{~B}$ & $38 \mathrm{~A}$ & $65 \mathrm{~A}$ & $54 \mathrm{~A}$ & $50 \mathrm{~A}$ & $38 \mathrm{~A}$ & $30 \mathrm{~A}$ & A 13 B & $3 \mathrm{C}$ \\
\hline $\begin{array}{l}\text { Vermelho } \\
\text { carmim }\end{array}$ & $\begin{array}{l}2,5 \mathrm{R} 5 / 12 \\
2,5 \mathrm{R} 5 / 10 \\
1,25 \mathrm{R} 5 / 14 \\
2,5 \mathrm{R} 4 / 14\end{array}$ & $2 \mathrm{C}$ & $2 \mathrm{C}$ & $3 \mathrm{C}$ & $2 \mathrm{C}$ & $3 \mathrm{~B}$ & $5 \mathrm{C}$ & $2 \mathrm{C}$ & $6 \mathrm{BC}$ & $12 \mathrm{~B}$ & $16 \mathrm{C}$ & $37 \mathrm{~A}$ & $45 \mathrm{~A}$ & $\begin{array}{l}43 \\
\mathrm{~A}\end{array}$ & $72 \mathrm{~A}$ & $61 \mathrm{~A}$ \\
\hline $\begin{array}{c}\text { Vermelho } \\
\text { sarmim-escuro }\end{array}$ & $\begin{array}{l}2,5 \mathrm{R} 4 / 10 \\
2,5 \mathrm{R} 4 / 12\end{array}$ & $0 \mathrm{C}$ & $0 \mathrm{C}$ & $0 \mathrm{C}$ & $0 \mathrm{C}$ & $0 \mathrm{~B}$ & $0 \mathrm{D}$ & $2 \mathrm{C}$ & $0 \mathrm{C}$ & $0 \mathrm{C}$ & $0 \mathrm{D}$ & $1 \mathrm{C}$ & $5 \mathrm{BC}$ & $\begin{array}{l}0 \\
\mathrm{~B}\end{array}$ & $9 \mathrm{~B}$ & $33 \mathrm{~B}$ \\
\hline CV\% & & 40,5 & 33,2 & 42,3 & 36,4 & 46,7 & 35,3 & 37,0 & 42,3 & 35,4 & 20,1 & 33,8 & 38,2 & 48,0 & 34,6 & 50,1 \\
\hline DM & & 9,7 & 8,2 & 10,4 & 9,1 & 11,6 & 8,9 & 9,3 & 11,1 & 8,5 & 5,0 & 8,3 & 9,5 & 11,8 & 8,3 & 11,8 \\
\hline
\end{tabular}

A avaliação da coloração das sementes após o teste de tetrazólio mediante comparação com as fichas de cor do catálogo de Munsell (Munsell, 1976) possibilitou a identificação precisa das cores apresentadas pelas sementes. Sugere-se a utilização desse catálogo em trabalhos de pesquisa sobre o teste de tetrazólio para padronizar e auxiliar na reprodução da metodologia, como uma forma de especificar a cor das sementes após a coloração pela solução de tetrazólio. Entretanto, não é recomendada a utilização dessa comparação na rotina da análise de sementes, pois é muito trabalhosa e pouco prática.

$\mathrm{Na}$ análise da correlação (Tabela 4) pode-se observar que houve correlação positiva e significativa entre os testes de germinação em areia e em papel, e também entre o teste de germinação em areia e o teste de tetrazólio na concentração de $0,075 \%$ por 180 e 240 minutos e de $0,2 \%$ por 120 minutos, e entre o teste de germinação em papel e o teste de tetrazólio na concentração de $0,2 \%$ por 60 e 120 minutos. Por esse motivo, estes tratamentos foram considerados os melhores, e qualquer um deles podem ser utilizados para a condução do teste de tetrazólio em sementes de mamoneira.

Apesar das recomendações existentes para sementes de mamoneira (Grabe, 1976; Brasil, 1992) prescreverem a utilização de solução de tetrazólio a 1,0\%, essa metodologia foi ineficiente neste estudo. Pesquisas realizadas com sementes de outras espécies também têm mostrado que soluções menos concentradas que as prescritas pelas Regras para Análise de Sementes (Brasil, 1992) têm possibilitado melhores resultados (Bittencourt e Vieira, 1997; França Neto et al., 1998; Dias e Barros, 1999; Santos et al., 2007; Bhering et al., 2005). 
TABELA 4. Correlação simples das médias dos testes de germinação em areia, em papel, e dos testes de tetrazólio (TZ) conduzidos nos tratamentos de concentração da solução e período de coloração, para sementes de mamoneira.

\begin{tabular}{|c|c|c|c|c|}
\hline \multicolumn{3}{|c|}{ Tratamentos } & Germinação em areia & Germinação em papel \\
\hline \multicolumn{3}{|c|}{ Germinação em papel } & $0,85 * *$ & --- \\
\hline \multirow{3}{*}{$\mathrm{TZ}$} & $0,075 \%$ & x $120 \mathrm{~min}$ & $-0,59^{\mathrm{ns}}$ & $-0,47^{\mathrm{ns}}$ \\
\hline & & x $180 \mathrm{~min}$ & $0,79^{*}$ & $0,73^{\mathrm{ns}}$ \\
\hline & & x $240 \mathrm{~min}$ & $0,85^{*}$ & $0,70^{\mathrm{ns}}$ \\
\hline \multirow{3}{*}{$\mathrm{TZ}$} & $0,1 \%$ & x $120 \mathrm{~min}$ & $0,11^{\mathrm{ns}}$ & $0,21^{\mathrm{ns}}$ \\
\hline & & x $180 \mathrm{~min}$ & $0,22^{\mathrm{ns}}$ & $0,66^{\mathrm{ns}}$ \\
\hline & & x $240 \mathrm{~min}$ & $-0,04^{\text {ns }}$ & $0,22^{\mathrm{ns}}$ \\
\hline \multirow{3}{*}{$\mathrm{TZ}$} & $0,2 \%$ & x $60 \mathrm{~min}$ & $0,74^{\mathrm{ns}}$ & $0,94 * *$ \\
\hline & & x $120 \min$ & $0,76^{*}$ & $0,96 * *$ \\
\hline & & x $180 \min$ & $-0,28^{\text {ns }}$ & $0,23^{\mathrm{ns}}$ \\
\hline \multirow{3}{*}{$\mathrm{TZ}$} & $0,5 \%$ & x $60 \mathrm{~min}$ & $0,23^{\mathrm{ns}}$ & $0,62^{\mathrm{ns}}$ \\
\hline & & $\mathrm{x} 90 \mathrm{~min}$ & $-0,12^{\mathrm{ns}}$ & $0,37^{\mathrm{ns}}$ \\
\hline & & x $120 \mathrm{~min}$ & $0,35^{\mathrm{ns}}$ & $0,68^{\mathrm{ns}}$ \\
\hline \multirow{3}{*}{$\mathrm{TZ}$} & $1,0 \%$ & x $30 \mathrm{~min}$ & $-0,62^{\mathrm{ns}}$ & $-0,13^{\mathrm{ns}}$ \\
\hline & & $\mathrm{x} \quad 60 \mathrm{~min}$ & $0,00^{\mathrm{ns}}$ & $0,46^{\mathrm{ns}}$ \\
\hline & & $\mathrm{x} \quad 90 \mathrm{~min}$ & $-0,43^{\mathrm{ns}}$ & $0,10^{\mathrm{ns}}$ \\
\hline
\end{tabular}

Considerando-se a eficiência na seleção dos lotes (Tabela 2), aliada à correlação com os testes de germinação em areia e em papel (Tabela 4), para a identificação do melhor tratamento de concentração da solução e período de coloração para o teste de tetrazólio, conclui-se que os melhores resultados foram obtidos com a imersão das sementes em solução de tetrazólio a $0,2 \%$ por 120 minutos. Neste tratamento a maioria das sementes apresentou-se nos grupos de cores rosa e rosa-escuro (Tabela 3), portanto essas podem ser consideradas como as cores características para o teste de tetrazólio em sementes de mamoneira, discordando de Grabe (1976), que considerou a cor vermelha como a característica nas sementes para esse teste nesta espécie.

\section{CONCLUSÕES}

Para avaliação do potencial fisiológico pelo teste de tetrazólio para sementes de mamoneira, as mesmas devem ser imersas na solução de tetrazólio na concentração de $0,2 \%$ por 120 minutos, na temperatura de $35^{\circ} \mathrm{C}$, para o desenvolvimento da coloração.

Após a coloração pela solução de tetrazólio a nomenclatura para as cores apresentadas pelas sementes viáveis de mamoneira são rosa e rosa-escuro.

\section{REFERÊNCIAS}

BANZATTO, D.A.; KRONKA, S.N. Experimentação agrícola. 4. ed. Jaboticabal: FUNEP/UNESP, 2006. 237p.

BARROS, D.I.; DIAS, D.C.F.S.; BHERING, M.M.; DIAS, L.A.S.; ARAÚJO, E.F. Uso do teste de tetrazólio para avaliação da qualidade fisiológica de sementes de abobrinha.

Revista Brasileira de Sementes, v.27, n.2, p.165-171, 2005.

BHERING, M.M.; DIAS, D.C.F.S.; BARROS, D.I. Adequação da metodologia do teste de tetrazólio para avaliação da qualidade fisiológica de sementes de melancia.

Revista Brasileira de Sementes, v.27, n.1, p.176-182, 2005.

BITTENCOURT, S.R.M.de.; VIEIRA, R.D. Use of reduced concentrations of tetrazolium solutions for the evaluation of the viability of peanut seed lots. Seed Science and Technology, v. 25, n. 1, p.75-82, 1997.

BRASIL. Ministério da Agricultura e Reforma Agrária. Secretaria Nacional de Defesa Agropecuária. Departamento Nacional de Produção Vegetal. Coordenação de Laboratório Vegetal. Regras para Análise de Sementes. Brasília, DF, 1992. 365 p. 
COIMBRA, R. de A.; TOMAZ, C. de A.; MARTINS, C.C.; NAKAGAWA, J. Testes de germinação com o acondicionamento dos rolos de papel em sacos plásticos. Revista Brasileira de Sementes, v.29, n.1, p. 92-97, 2007.

DELOUCHE, J.C.; STILL, T.W.; RASPET,M.; LIENHARD, M. O teste de tetrazólio para viabilidade da semente. Brasília, DF: AGIPLAN, 1976. 103p.

DIAS, M.C.L.L.; BARROS, A.S.R. Avaliação da qualidade de sementes de milho. Londrina: IAPAR, 1995. 43p. (IAPAR. Circular, 88).

DIAS, M.C.L.L.; BARROS, A.S.R. Metodologia do teste de tetrazólio em sementes de milho. In: KRZYZANOWSKI, F.C.; VIEIRA, R.D.; FRANÇA NETO, J.B. (Ed.). Vigor de sementes: conceitos e testes Londrina: ABRATES, 1999. cap. 8.4, p.1-10.

FRANÇA NETO, J.B. Testes de tetrazólio para determinação do vigor de sementes. In:. KRZYZANOWSKI, F.C.; VIEIRA, R.D.; FRANÇA NETO, J.B. Vigor de sementes: conceitos e testes Londrina: ABRATES, 1999. cap.8, p. 1-7.

FRANÇA NETO, J.B.; KRZYZANOWSKI, F.C.; COSTA, N.P. da. O teste de tetrazólio em sementes de soja. Londrina: EMBRAPA-CNPSo, 1998. 72p. (EmbrapaCNPSo. Documentos, 116).

GASPAR OLIVEIRA, C.M. Avaliação da qualidade fisiológica de sementes de mamoneira (Ricinus communis L.) pelo teste de tetrazólio. 2007, 96f. Tese (Doutorado em Agronomia) - Faculdade de Ciências Agronômicas Universidade Estadual Paulista, Botucatu, 2007.

GASPAR OLIVEIRA, C.M.; MARTINS, C.C.; NAKAGAWA, J. Germination test improvement for castor bean seeds (Ricinus communis L.) In: CONGRESSO BRASILEIRO DE SEMENTES, 15., 2007, Foz do Iguaçu. Anais... Foz do Iguaçu, 2007. p.70.

GASPAR OLIVEIRA， C.M.; MARTINS， C.C.; NAKAGAWA, J. Método de preparo das sementes de mamoneira (Ricinus communis L.) para o teste de tetrazólio.
Revista Brasileira de Sementes, v.31, n.1, p.160-167, 2009.

GRABE, D.F. Manual do teste de tetrazólio em sementes. Brasília, DF: AGIPLAN. 1976. 86p.

MAGUIRE, J.D. Speed of germination-aid in selection and evaluation for seedling emergence and vigor. Crop Science, v. 2, n.2, p. 176-177, 1962.

MARCOS FILHO, J.; CÍCERO, S.M.; SILVA, W.R. Avaliação da qualidade das sementes. Piracicaba: FEALQ, 1987. 230p.

MOORE, R.P. Handbook on tetrazolium testing. Zurich: International Seed Testing Association, 1985. 99p.

MUNSELL, A. H. Munsell book of color. Baltimore: macbeth division of kollmorgen, 1976. (Mathefinish collection).

PADRÕES para produção e comercialização de sementes de mamona cultivares híbridas. Diário Oficial [da] República Federativa do Brasil, Brasília, DF, n. 243. 20 de dez. de 2005. Seção 1. Disponível em: <http://www.seab.pr.gov. br/arquivos/File/PDF/padroes_mamona.pdf>Acesso em: 20 maio 2006.

SANTOS, M.A.O.; NOVEMBRE, A.D.L.C.; MARCOS FILHO, J. Tetrazolium test to assess viability and vigour of tomato seeds. Seed Science and Technology, v. 35, n. 1, p. 213-223, 2007.

VIEIRA, M.G.G.C.; VON PINHO.; E.V.R. Metodologia do teste de tetrazólio em sementes de algodão. In: KRZYZANOWSKI, F.C.; VIEIRA, R.D.; FRANÇA NETO, J.B. (Ed.). Vigor de sementes: conceitos e testes. Londrina: ABRATES, 1999. cap. 8.1, p. 1-13.

WETZEL, M.M.V.da S.; CÍCERO, S.M.; FERREIRA, B.C. da S. Aplicação do teste de tetrazólio em sementes de seringueira. Revista Brasileira de Sementes, v.14, n.1, p.83-88, 1992. 